\title{
Transfers to distant periodic orbits around the Moon via their invariant manifolds
}

\author{
G. Mingotti ${ }^{a}$, F. Topputo ${ }^{\text {b,* }}$, F. Bernelli-Zazzera ${ }^{\mathrm{b}}$ \\ a Institut für Industriemathematik, Universität Paderborn, Warburger Str. 100, 33098 Paderborn, Germany

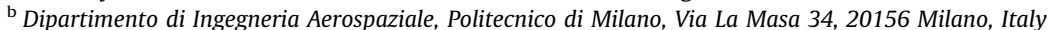

\section{A R T I C L E I N F O}

\section{Article history:}

Received 5 August 2011

Received in revised form

9 April 2012

Accepted 12 April 2012

Keywords:

Restricted three-body problem

Invariant manifolds

Low-energy transfers

Dynamical system theory

Low-thrust propulsion

\begin{abstract}
A B S T R A C T
This paper presents two ways to transfer a spacecraft to distant periodic orbits in the Earth-Moon system. These unstable periodic orbits of the restricted three-body problem reveal a rich phase-portrait structure that can be used by space missions. Through the perspective of dynamical system theory, distant periodic orbits' invariant manifolds can be exploited to design novel low-energy trajectories in the Earth-Moon framework. Interior and exterior transfers are presented. The latter use impulsive, highthrust propulsion to target the stable manifold from the exterior. Interior transfers are instead formulated with continuous, low-thrust propulsion. The attainable sets are used in both cases to handle families of either coast arcs or low-thrust orbits. First guess solutions are optimized in the framework of the Sun-Earth-Moon-Spacecraft restricted four-body problem through direct transcription and multiple shooting. The novelty of the presented solutions, as well as their efficiency, is demonstrated through examples.
\end{abstract} (c) 2012 Elsevier Ltd. All rights reserved.

\section{Introduction}

Unstable orbits analysis shows many opportunities, from purely theoretical speculations to practical applications [24]. Unstable orbits were first found by numerically exploring the Hill's limiting case of the restricted threebody problem by Hénon [9], who systematically studied and classified symmetrical, simple-periodic orbits (i.e., orbits that are symmetric with respect to the $x$-axis and intersect it only twice). As the Hill's problem admits an integral of motion, $\Gamma$, the orbits are organized into oneparameter families. Families $a, c, f, g, g^{\prime}$ were found, and

\footnotetext{
Part of the work described in this paper has been presented at the 20th AAS/AIAA Space Flight Mechanics Meeting San Diego (CA), USA, 14-17 February, 2010.

*Corresponding author. Tel.: +3902 2399 8640; fax: +39 022399 8334.

E-mail addresses: giorgio.mingotti@mail.polimi.it (G. Mingotti), topputo@aero.polimi.it (F. Topputo), bernelli@aero.polimi.it (F. BernelliZazzera).
}

their stability was studied. Among these, orbits belonging to the $g$-family are of interest in this work. These orbits can be continued into the restricted three-body problem (by using the mass ratio as continuation parameter) staring from the initial conditions in [9] and solving a two-point boundary value problem at each step. More specifically, a subset of the g-type orbits is considered in this paper for which $\Gamma<4.99986$ and the stability index is greater than one (see Table 4 in [9]). As the g-orbits originate from the central orbits around the smaller primary, they are stable in the region close to the smaller primary, though their stability index continuously varies when $\Gamma$ decreases (and the orbit's size increases accordingly). In particular, for $\Gamma<4.99986$ the $g$-orbits become unstable. Since this condition is reached when portions of the orbits lie far from the smaller primary (farther then the two equilibrium points close to it), these orbits are labeled as distant periodic orbits (DPOs). This terminology is used in the reminder.

From the astrodynamics perspective, DPO belonging to the Earth-Moon restricted three-body problem are of 
interest. If combined with the Sun-Earth restricted threebody problem, Earth-Moon DPO may define low-energy trajectories from the Earth's vicinity to the Moon's neighborhood and return. This can be done in a deterministic fashion [13]. Using DPO's intrinsic dynamics to design low-energy lunar transfers mimics the technique already established in literature, with the exception that the invariant manifolds associated to the DPO are exploited in place of those associated to the libration point orbits $[8,10,11,14,15]$. This approach is also used in Mingotti and Gurfil [18] to design transfers to DPO around Mars (with and without lunar gravity assists).

In the present study, two types of transfers to DPO in the Earth-Moon system are presented. These two strategies differ in the kind of propulsion considered, which in turn affects the structure of the transfers as well as their geometry. In both cases, the concept of stable manifold associated to DPO is used: in order to reach the final periodic orbit, the spacecraft has to be placed, in the phase space sense, on the stable manifold of that orbit. This requires handling a global representation of the stable manifold. In the RTBP, these can be determined by backward integrating the periodic orbit perturbed along the stable eigendirection [7,27]. Once placed on the stable manifold, the dynamical system provides at bringing the spacecraft to the final orbit at zero cost. To do this, it is important to assess whether or not the stable manifold associated with the final DPO approaches the Earth, or, more specifically, the departure orbit. It can be shown that the invariant manifolds associated with DPO around the Moon do not approach the Earth [17]. For this reason, the focus is to maneuver the spacecraft (starting from a prescribed Earth orbit) such that the DPO stable manifold is reached. The stable manifold is made up by two branches, and each of them can be targeted. In this perspective, it would be desirable to choose the propulsion system that better fits the branch of the manifold to target. In particular, using the low-thrust propulsion to reach the exterior branch is not deemed a viable option as it would require long transfer times and high propellent consumptions. Thus, we consider the high-thrust propulsion to reach the exterior branch of the stable manifold. The low-thrust propulsion is instead considered in the interior branch case. This is deemed more efficient than possible two-impulse transfers associated to high-thrust propulsion. In summary, the two following options are considered, depending on the propulsion used.

Low-thrust, interior transfers to DPO. Low-thrust solutions are found by targeting a piece of the interior branch of the stable manifolds of the DPO. This is done by spiraling around the Earth up to reach a point that lies on the stable manifold. This strategy recalls that already formulated to design low-thrust transfers to halo orbits in the Earth-Moon system [14].

Single-impulse, exterior transfers to DPO. Impulsive transfers to DPO are found with the coupled restricted three-body problem approximation $[8,11]$. In this framework, two pieces (one defined in the Sun-Earth problem and the other in the Earth-Moon problem) are patched together to define the whole transfer trajectory [15]. The originality of such approach consists in using, in the second part of the transfer, the stable manifold of the DPO, so extending the approach based on the exploitation of the Lyapunov orbits' stable manifolds.

In both cases, attainable sets are introduced to handle families of orbits at once. This includes both thrust and coast arcs, with the latter thought as propelled orbits with zero thrust. This approach generalizes the concept of attainable set with respect to previous works where only tangential thrust orbits where considered [19,20,22]. Attainable sets manipulation allows us to defined accurate first guess solutions that are later optimized in a more refined gravitational model by means of a direct transcription and multiple shooting procedure.

Summarizing, the purposes of the work are:

- to extend the design technique proposed by Koon et al. [11] and further developed by Mingotti et al. [15] (by including low-thrust propulsion) to the case of final DPO orbits around the Moon;

- to design low-thrust, stable-manifold interior trajectories to final DPO around the Moon in analogy with low-thrust transfers to halos [14].

The remainder of the paper is organized as follows. The dynamical models are recalled, and the statement of the problems are given in the first part of the paper. In the second part, the trajectory design strategy is described by defining the attainable sets. The optimization problem is formulated in the third part where sample optimal solutions are also shown.

\section{Background}

This section summarizes all the notions underlying the present work. As the main scope of the paper is to design transfers to DPO around the Moon, the background material is defined by using references, and going into details would lead us beyond the scopes of the paper. The reader can consult references for a compete derivation of the equations of motion and distant periodic orbits.

\subsection{The planar circular restricted three-body problem}

The motion of the spacecraft, $m_{3}$, is studied in the gravitational field generated by the mutual circular motion of two primaries of masses $m_{1}, m_{2}$, respectively, about their common center of mass (see Fig. 1(a)). It is assumed that $m_{3}$ moves in the same plane of $m_{1}, m_{2}$ under the following equations [26]:

$\ddot{x}-2 \dot{y}=\frac{\partial \Omega}{\partial x}, \quad \ddot{y}+2 \dot{x}=\frac{\partial \Omega}{\partial y}$,

where the auxiliary function is

$\Omega(x, y, \mu)=\frac{1}{2}\left(x^{2}+y^{2}\right)+\frac{1-\mu}{r_{1}}+\frac{\mu}{r_{2}}+\frac{1}{2} \mu(1-\mu)$,

and $\mu=m_{2} /\left(m_{1}+m_{2}\right)$ is the mass parameter of the threebody problem. Eqs. (1) are written in a barycentric rotating frame with nondimensional units: the angular velocity of $m_{1}, m_{2}$, their distance, and the sum of their masses are all set to the unit value. It is easy to verify that 
a

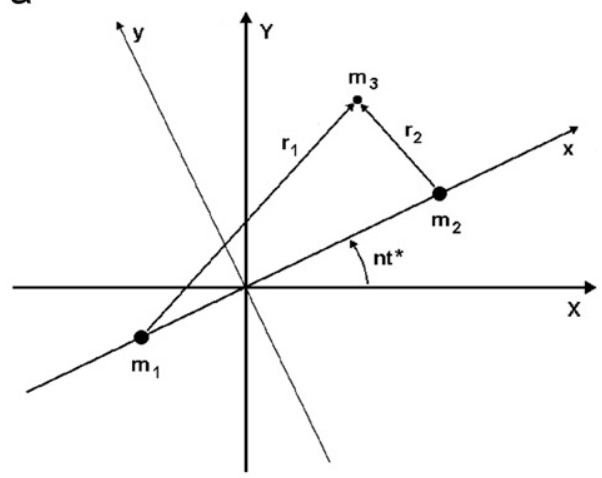

b

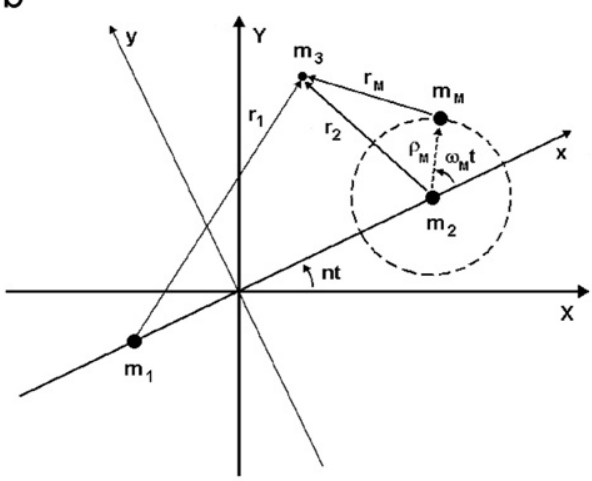

Fig. 1. Mathematical models used to catch the physics of the problem. (a) Planar circular restricted three-body problem. (b) Moon-perturbed SunEarth RFBP.

the primary of mass $1-\mu$, is located at $(-\mu, 0)$, whereas the smaller primary $\mu$, is located at $(1-\mu, 0)$; thus, the distances between $m_{3}$ and the primaries in Eq. (2) are

$r_{1}=\left[(x+\mu)^{2}+y^{2}\right]^{1 / 2}, \quad r_{2}=\left[(x+\mu-1)^{2}+y^{2}\right]^{1 / 2}$.

For fixed $\mu$, the Jacobi integral reads

$J(x, y, \dot{x}, \dot{y})=2 \Omega(x, y, \mu)-\left(\dot{x}^{2}+\dot{y}^{2}\right)$,

and, for a given energy $C$, it defines a three-dimensional manifold

$F(C)=\left\{(x, y, \dot{x}, \dot{y}) \in \mathcal{R}^{4} \mid J(x, y, \dot{x}, \dot{y})-C=0\right\}$.

The projection of $F(C)$ on the configuration space $(x, y)$ defines the Hill's curves bounding the allowed and forbidden regions associated with prescribed values of $C$. The vector field defined by Eq. (1) has five well-known equilibrium points, known as the Lagrange points, $L j$, $j=1, \ldots, 5$. There exists a family of retrograde Lyapunov orbits around $L 1, L 2$, and two-dimensional stable and unstable manifolds emanating from them [3,12].

The system governed by Eq. (1) is used alternatively to describe the motion of the spacecraft either in the SunEarth (SE) or in the Earth-Moon (EM) system. The mass parameter value assumed for these models are $\mu_{S E}=$ $3.0034 \times 10^{-6}$ and $\mu_{E M}=1.2150 \times 10^{-2}$, respectively.

As for the SE model, the generic periodic orbit about $L j$, $j=1,2$, is referred to as $\gamma_{j}$, whereas its stable and unstable manifolds are labeled $W^{s}\left(\gamma_{j}\right), W^{u}\left(\gamma_{j}\right)$. In the EM model, the generic periodic orbit about $L j, j=1,2$, is called $\lambda_{j}$, while its stable and unstable manifolds are named $W^{s}\left(\lambda_{j}\right)$, $W^{u}\left(\lambda_{j}\right)$.

\subsection{The Moon-perturbed Sun-Earth restricted three-body problem}

When the gravitational attraction of the Moon is considered in the SE model, a bicircular restricted fourbody problem (RFBP) is defined (see Fig. 1(b)). To derive the equations of this model, the following assumptions are considered, recalling that the orbits of the primaries show low eccentricities $(\simeq 0.01, \simeq 0.05$, for the Earth,
Moon, respectively), and the Moon inclination on the ecliptic is small $\left(\simeq 5^{\circ}\right)$ :

- the Sun and the Earth move on circular orbits around their common center of mass;

- the Earth-Moon barycenter moves on a circular orbit around the center of mass of the Sun-Earth-Moon system.

Under these assumptions, the equations of motion of the spacecraft are [22]

$\ddot{x}-2 \dot{y}=\frac{\partial \Omega_{M}}{\partial x}, \quad \ddot{y}+2 \dot{x}=\frac{\partial \Omega_{M}}{\partial y}, \quad \dot{\theta}=\omega_{M}$,

where the auxiliary function is

$\Omega_{M}(x, y, \theta)=\Omega\left(x, y, \mu_{S E}\right)+\frac{m_{M}}{r_{M}}-\frac{m_{M}}{\rho_{M}^{2}}(x \cos \theta+y \sin \theta)$.

The dimensionless physical constants introduced to describe the Moon influence are coherent with those of the SE model. Thus, the distance between the Moon and the Earth is $\rho_{M}=2.5721 \times 10^{-3}$, the mass of the Moon is $m_{M}=3.6942 \times 10^{-8}$, and its angular velocity with respect to the SE rotating frame is $\omega_{M}=1.2367 \times 10^{1}$. The location of the Moon is at $\left(1-\mu_{S E}+\rho_{M} \cos \theta, \rho_{M} \sin \theta\right)$, such that the Spacecraft-Moon distance is

$r_{M}=\left[\left(x-1+\mu_{S E}-\rho_{M} \cos \theta\right)^{2}+\left(y-\rho_{M} \sin \theta\right)^{2}\right]^{1 / 2}$.

\subsection{Distant periodic orbits}

In the framework of Hill's problem $(\mu \rightarrow 0)$ there exist periodic orbits. Due to the existence of the integral of motion, these orbits are grouped in one-parameter families, each family containing a simple infinity of periodic orbits, whose properties vary continuously from one end of the family to the other. For nonzero mass parameters, a transformation is needed to obtain these orbits in the RTBP from those available [9].

Theory predicts, however, that there are infinitely many families of periodic orbits. The simplest of them are those symmetric with respect to the $x$-axis, and 
a

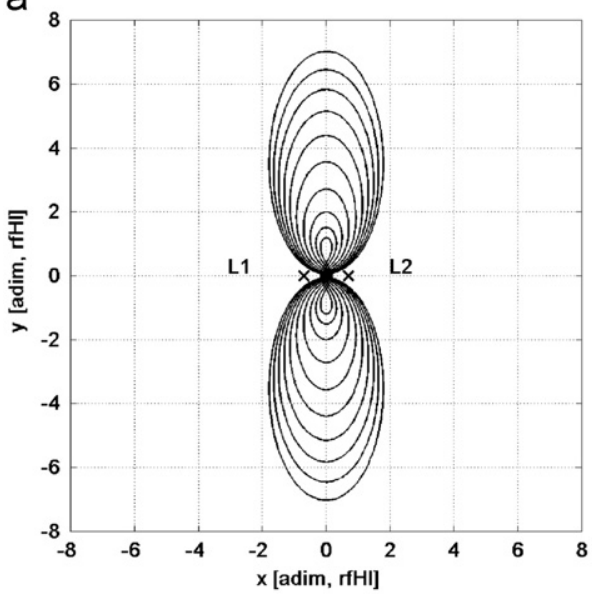

b

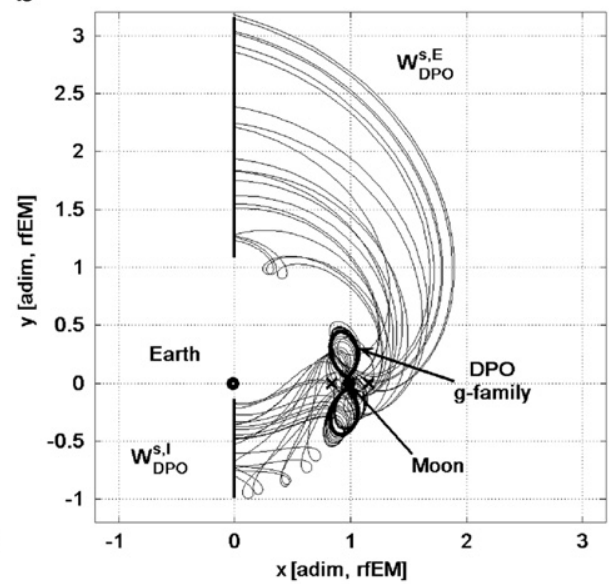

Fig. 2. Distant periodic orbits belonging to the $g$-family [9] and their invariant manifolds. In (b), both the interior branch $(I)$ and the exterior branch ( $E$ ) of the stable manifold $W_{D P O}^{s}$ are drawn (DPO shown bold). (a) Family $g$ of DPO in the Hill's problem (rotating frame). (b) A g-type orbit and its stable manifold in the Earth-Moon system (rotating frame).

simple-periodic; i.e., intersecting the $x$-axis only twice. According to Hénon nomenclature [9], there are five groups. Orbits of families $a$ and $c$ originating as libration point orbits, are all unstable. Orbits of family $f$, originating as retrograde orbits about the smaller primary, are all stable. Orbits of families $g$ and $g$ ', originating as orbits only around the smaller primary, are stable in some intervals. These orbits are computed numerically from the initial conditions in Hénon [9]. A numerical continuation procedure, implementing $\mu$ as continuation parameter, together with a simple shooting algorithm, delivers DPO in the RTBP with good accuracy (see Fig. 2).

The orbits belonging to the unstable g-family show an interesting behavior as they possess local stable and unstable manifolds that can be globally extended. The structure of these manifolds define subsets that may provide free transport channels (see Fig. 2(b)). From a mission design point of view, a spacecraft can travel naturally to or from unstable periodic orbits along their stable or unstable manifolds, respectively [23]. This complex structure intersects many interesting regions in the RTBP: the small primary neighborhood and the libration point vicinity.

\section{Attainable sets and low-thrust propulsion}

A general approach useful to handle coast arcs, lowthrust arcs, and invariant manifolds is proposed. This formulation is based on a perturbed version of the RTBP. The perturbation is the low-thrust propulsion, whose profile over time can be designed to achieve different types of transfer arcs, as well as several final conditions at Moon arrival.

To model the controlled motion of $m_{3}$ under both the gravitational attractions of $m_{1}, m_{2}$, and the low-thrust propulsion, the following differential equations are considered:

$\ddot{x}-2 \dot{y}=\frac{\partial \Omega}{\partial x}+\frac{T_{x}}{m}, \quad \ddot{y}+2 \dot{x}=\frac{\partial \Omega}{\partial y}+\frac{T_{y}}{m}, \quad \dot{m}=-\frac{T}{I_{s p} g_{0}}$, where $T=\sqrt{T_{x}^{2}+T_{y}^{2}}$ is the thrust magnitude, $I_{s p}$ the engine's specific impulse, and $g_{0}$ the gravitational acceleration at sea level [15]. The controlled RTBP can be seen as a special Hamiltonian system with dissipation [2]. The equations refer to the planar, circular, restricted threebody problem, expressed in a synodic reference frame rotating with the angular velocity of the primaries.

The thrust law $\mathbf{T}(t)=\left\{T_{x}(t), T_{y}(t)\right\}^{\top}, t \in\left[t_{i}, t_{f}\right]$, has to be determined by solving an optimal control problem $\left(t_{i}\right.$ and $t_{f}$ are the initial and final time, respectively). However, in order to build first guess solutions, the profile of $\mathbf{T}$ over time is prescribed at this stage. In particular, using tangential thrust, attainable sets can be defined in the same fashion as reachable sets are defined by Dellnitz et al. [4].

Let $\mathbf{y}_{i}=\left\{x_{i}, y_{i}, \dot{x}_{i}, \dot{y}_{i}, m_{i}\right\}^{\top}$ be a vector representing a generic initial state, and let the flow of system of Eq. (9) at time $t$ be $\phi_{\mathbf{T}(\tau)}\left(\mathbf{y}_{i}, t_{i} ; t\right)$, starting from $\left(\mathbf{y}_{i}, t_{i}\right)$ and considering the thrust profile $\mathbf{T}(\tau), \tau \in\left[t_{i}, t\right]$. With this notation, it is possible to define the generic point of a tangential lowthrust trajectory through

$\mathbf{y}(t)=\phi_{\overline{\mathbf{T}}}\left(\mathbf{y}_{i}, t_{i} ; t\right)$,

where $\overline{\mathbf{T}}=\bar{T}(\mathbf{v} / v), v=\sqrt{\dot{x}^{2}+\dot{y}^{2}}, \mathbf{v}=\{\dot{x}, \dot{y}\}^{\top}$. Eq. (10) represents the flow of the differential system of Eq. (9), when constant tangential thrust of magnitude $\bar{T}$ is considered (see Mingotti et al. [16] for a comparison between tangential thrust in either rotating or inertial frame is shown). The low-thrust orbit, at time $t$, can be expressed as

$\gamma_{\overline{\mathbf{T}}}\left(\mathbf{y}_{i}, t\right)=\left\{\phi_{\overline{\mathbf{T}}}\left(\mathbf{y}_{i}, t_{i} ; \tau\right) \mid \tau \leq t\right\}$,

where the dependence on the initial state $\mathbf{y}_{i}$ is kept. The attainable set, at time $t$, can be defined as

$\mathcal{A}_{\overline{\mathbf{T}}}(t)=\bigcup_{\mathbf{y}_{i} \in \mathcal{Y}} \gamma_{\overline{\mathbf{T}}}\left(\mathbf{y}_{i}, t\right)$,

where $\mathcal{Y}$ is a domain of admissible initial conditions. Attainable set in Eq. (12) is associated with a generic $\mathcal{Y}$; 
this set can be defined for the different types of transfers. Thanks to the definition of $\mathcal{A}_{\overline{\mathrm{T}}}(t)$, low-thrust propulsion can be incorporated in a three-body frame, using the same methodology developed for the invariant manifolds [11]. See Mingotti et al. [19,20,22] for further features and applications of attainable sets. The attainable sets formalism can be adapted to design either low-thrust transfer to the Moon or single-impulse stable manifold trajectories to lunar DPO. For the latter it is important to note that attainable sets associated to coast arcs are defined by simply setting $\mathbf{T}=0$ in Eq. (12).

\section{Earth escape stage}

In this section, the first part of the transfers is described. As for the interior low-thrust stable manifold trajectories, the Earth escape stage is performed through a continuous low-thrust spiral orbit, defined in the EarthMoon RTBP. Pertaining the exterior transfers, the Earth escape trajectory is achieved by an impulsive maneuver that places the spacecraft on a translunar orbit that flies in the proximity of the Sun-Earth invariant manifolds.

\subsection{Low-thrust escape}

In the low-thrust, stable manifold transfers to DPO around the Moon, the Earth escape stage is defined as follows. The spacecraft is assumed to be initially on a Geostationary Transfer Orbit (GTO) about the Earth, although the formulation is valid for any initial orbit with given eccentricity, $e$, and periapsis radius $r_{p}$. The orientation of this orbit, $\omega_{E}$, is not fixed (see Fig. 3(a)). The transfer begins when the spacecraft is at the perigee. From this initial point on, the spacecraft can use the lowthrust system to raise the orbit as shown in Fig. 3(b).

The initial state, $\mathbf{y}_{i}$, corresponds to the periapsis point of departure orbit. As both eccentricity and apsidal altitudes are prescribed, this initial state depends only upon the argument of perigee; i.e. $\mathbf{y}_{i}=\mathbf{y}_{i}\left(\omega_{E}\right)$. The domain of admissible initial states can be written as

$\mathcal{Y}^{E}=\left\{\mathbf{y}_{i}\left(\omega_{E}\right) \mid \omega_{E} \in[0,2 \pi]\right\}$, and therefore the attainable set at time $t$ of interior Earthescape orbits, $\mathcal{E}(I / \overline{\mathbf{T}})(t)$, is

$\mathcal{E}_{\overline{\mathbf{T}}}^{I}(t)=\bigcup_{\mathbf{y}_{i} \in \mathcal{Y}^{E}} \gamma_{\overline{\mathbf{T}}}\left(\mathbf{y}_{i}\left(\omega_{E}\right), t\right)$.

It is important reminding that the time $t$ in Eq. (14) stands for the duration of tangential low-thrust. Typically, for short times the low-thrust is not able to sufficiently raise the initial orbit, while for long times the orbit energy is increased too much. The attainable set in Eq. (14) has to be searched within the time interval $t \in\left[0, T_{E}\right]$, where $T_{E}$ is a certain prescribed upper bound for the duration of the low-thrust portion.

\subsection{Single-impulse escape}

In low-energy, exterior transfers to lunar DPO, the spacecraft is assumed to be initially on a circular orbit around the Earth at an altitude $h_{E}$. An impulsive maneuver, $\Delta v_{E}$, places the spacecraft on a translunar trajectory. From this point on, no maneuvers are admitted, and the spacecraft has to achieve capture at the Moon at zero cost.

In the $S E$ model, a Jacobi constant $C_{S E} \lesssim C_{2}$ assures that both $\gamma_{1}$ and $\gamma_{2}$ exist together with their stable and unstable manifolds, $\gamma_{2}, W^{s}\left(\gamma_{2}\right)$ and $W^{u}\left(\gamma_{2}\right)$, respectively. To exploit the structure of both $W^{s}\left(\gamma_{2}\right)$ and $W^{u}\left(\gamma_{2}\right)$, two surfaces of section are introduced to study their cuts. Section $S_{A}$, making an angle $\varphi_{A}$ (clockwise) with the $x$-axis and passing through the Earth, is considered to cut $W^{S}\left(\gamma_{2}\right)$, whereas section $S_{B}$, inclined by $\varphi_{B}$ (counterclockwise) on the $x$-axis and passing through the Earth, is assumed for $W^{u}\left(\gamma_{2}\right)$ (see Fig. 4(a)). The corresponding section curves, $\partial \Gamma_{2}^{s}, \partial \Gamma_{2}^{u}$, represented on the $\left(r_{2}, \dot{r}_{2}\right)$-plane, are diffeomorphic to circles (in Fig. 4(b), $r_{2}=y, \dot{r}_{2}=\dot{y}$ as $x=1-\mu$, $\left.\varphi_{A}=\varphi_{B}=\pi / 2\right)$.

The Earth-escape set, $\mathcal{E}_{S E}^{E}$, obtained in the SE model, uses orbits that are close to both $W^{s}\left(\gamma_{2}\right)$ and $W^{u}\left(\gamma_{2}\right)$, and that, at the same time, intersect the departure orbit. This piece of trajectory is the same used to construct exterior Earth-Moon transfers. The reader can refer to Mingotti et al. [15] for a detailed derivation of $\mathcal{E}_{S E}^{E}$, and to Mingotti et al. [22] for the inclusion of lunar gravity assists. a

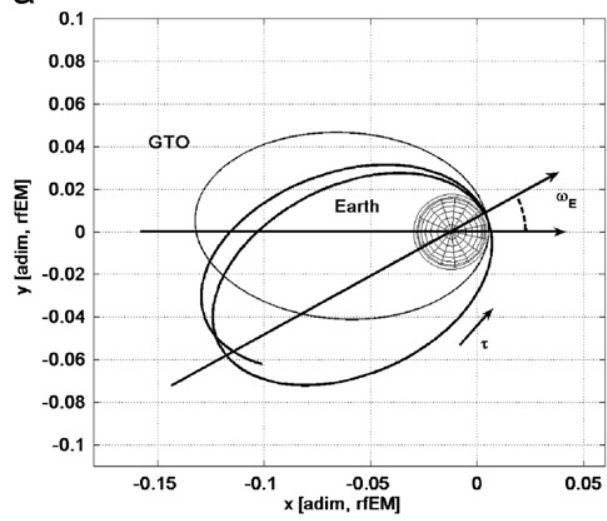

b

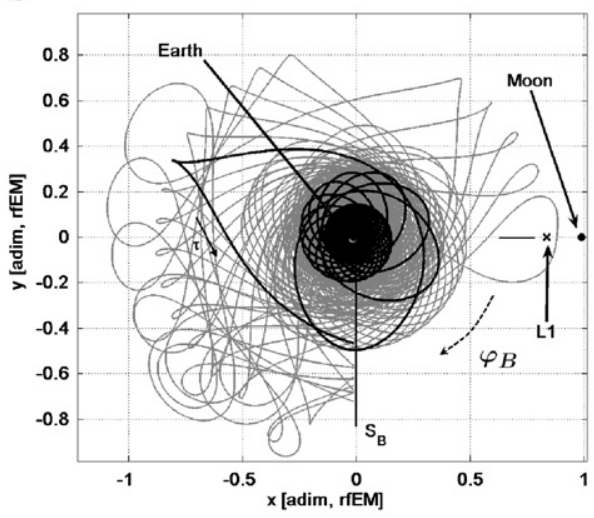

Fig. 3. Initial orbit and low-thrust escape trajectory. (a) Initial transfer orbit. (b) Low-thrust Earth escape trajectory. 
a

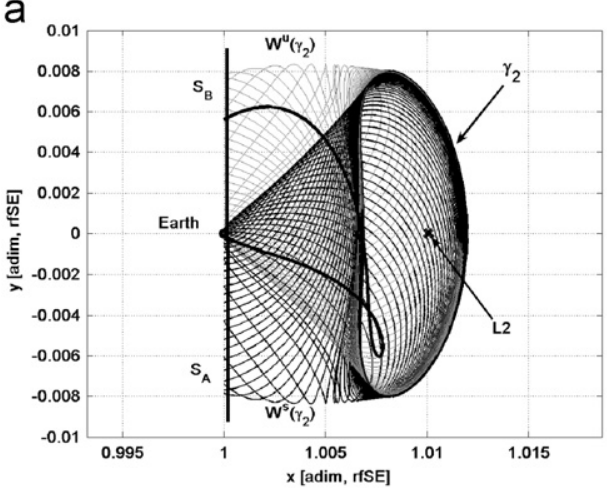

b

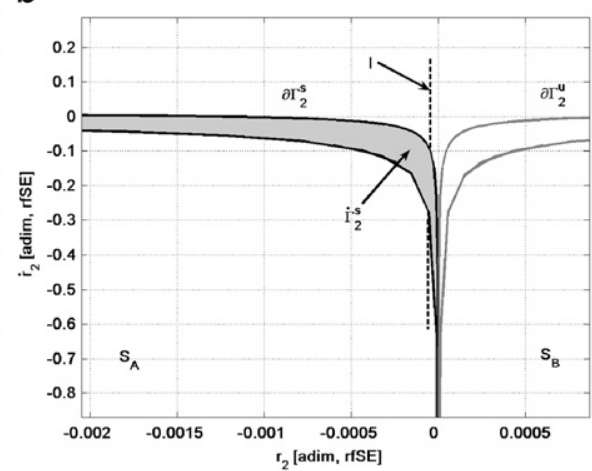

Fig. 4. Stable and unstable manifolds $W^{s}\left(\gamma_{2}\right), W^{u}\left(\gamma_{2}\right)$ associated with the $L 2$ Lyapunov orbit $\gamma_{2}$, and their section curves $\partial \Gamma_{2}^{s}$, $\partial \Gamma_{2}^{u}$, respectively. In (a), the bold line stands for a sample Earth escape trajectory. In (b), the set $\dot{\Gamma}_{2}^{s}$ (gray) is made up by the points of $S_{A}$ that lie inside $\partial \Gamma_{2}^{s}$, whereas the line $l$ (dashed) is the locus of points being at $h_{E}=167 \mathrm{~km}$ altitude above the Earth surface. (a) $W^{s}\left(\gamma_{2}\right)$ and $W^{u}\left(\gamma_{2}\right)$. (b) $\partial \Gamma_{2}^{s}, \dot{\Gamma}_{2}^{s}$, and $\partial \Gamma_{2}^{u}$ sets.

a

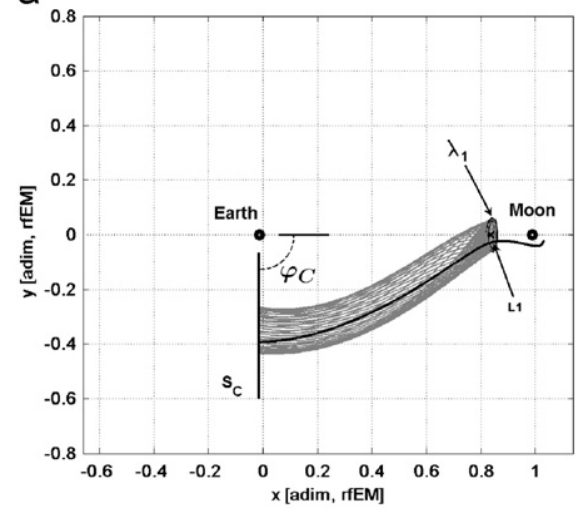

b

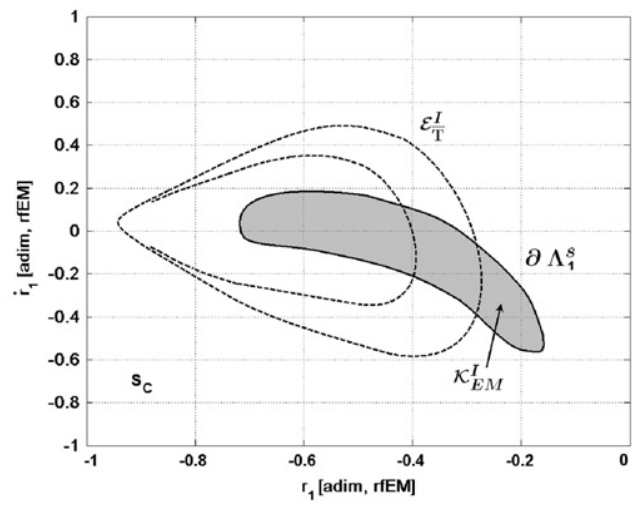

Fig. 5. Stable manifold $W^{s}\left(\lambda_{1}\right)$ and its section curve $\partial \Lambda_{1}^{s}$. The latter is used to define the set of orbits that lead to Moon capture $\mathcal{K}_{E M}^{I}$ (gray area). The Earth low-thrust escape set $\mathcal{E}(I / \mathbf{T})(t)$ defined in Section 4.1 is reported in (a). (a) Interior branch of $W^{s}\left(\lambda_{1}\right)$. (b) $\mathcal{E}(I / \overline{\mathbf{T}})(t)$ and $\mathcal{K}_{E M}^{I}$ sets.

\section{Stable manifold capture at the Moon}

\subsection{Interior capture}

In the EM model, if the energy is restricted to $C_{E M} \lesssim C_{1}$, $\lambda_{1}$ exists, and the Hill's regions are opened at $L 1$. To consider a capture via $L 1, W^{S}\left(\lambda_{1}\right)$ is computed. Section $S_{C}$, making an angle $\varphi_{C}$ (clockwise) with the $x$-axis and passing through the Earth, is considered to cut $W^{s}\left(\lambda_{1}\right)$. The corresponding section curve, $\partial \Lambda_{1}^{s}$ is represented in the $\left(r_{1}, \dot{r}_{1}\right)$-plane. The set $\mathcal{K}_{E M}^{I}=\dot{\Lambda}_{1}^{s}$ is defined, being $\dot{\Lambda}_{1}^{s} \in$ $S_{C}$ the set of points inside $\partial \Lambda_{1}^{s}$, as the set that leads to lunar capture from interior (see Fig. 5).

Let us now consider a g-type DPO, with $J=$ 3.00002258271 in the EM system (Fig. 6(a)). Its stable manifold does not reach the Earth at a sufficiently low altitude to permit a direct insertion from a parking orbit. When a continuously propelled arc is introduced, it is possible to raise the initial orbit to place the spacecraft on the DPO stable manifold [14]. Thus, the initial part of the transfer is an orbit belonging to $\mathcal{E}(I / \overline{\mathbf{T}})(t)$. The second part of the transfer is made up by ballistic orbits that reach the final DPO. These orbits can be thought as a special attainable set with $T=0$ (no thrust). The transfer ends when the spacecraft reaches a point of the final DPO about the Moon. This orbit is assumed fixed and chosen according to prescribed mission requirements (e.g., the period, the altitude with respect to the Moon surface, etc). In details, the final state of the transfers, $\mathbf{y}_{f}$, can be any point that belongs to the DPO (insertion point, $\mathbf{y}_{I N S}$, in Fig. 6(a)). The generic insertion point is found by flowing the initial nominal point $\mathbf{y}_{i}$ for a time $\tau_{M} \leq P_{D P O}$, being $P_{D P O}$ the DPO's period

$\mathbf{y}_{f}=\mathbf{y}\left(\tau_{M}\right)=\phi\left(\mathbf{y}_{i}, 0 ; \tau_{M}\right)$.

The domain of admissible final states is then written as follows:

$\mathcal{Y}^{M}=\left\{\mathbf{y}_{f}\left(\tau_{M}\right) \mid \tau_{M} \in\left[0, P_{D P O}\right]\right\}$,

and the attainable set, for some $t \geq 0$ (i.e., $-t$ is a backward integration), containing stable manifold trajectories is

$\mathcal{C}_{\mathbf{w}}^{I}(-t)=\bigcup_{\mathbf{y}_{f} \in \mathcal{Y}^{M}} \gamma_{\mathbf{w}}\left(\mathbf{y}_{f}\left(\tau_{M}\right),-t\right)$. 
a

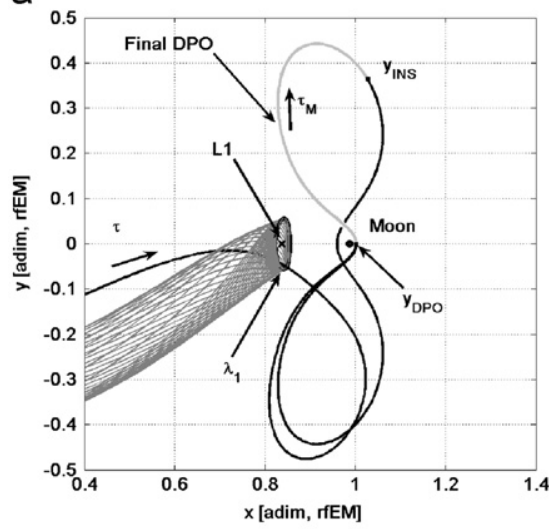

b

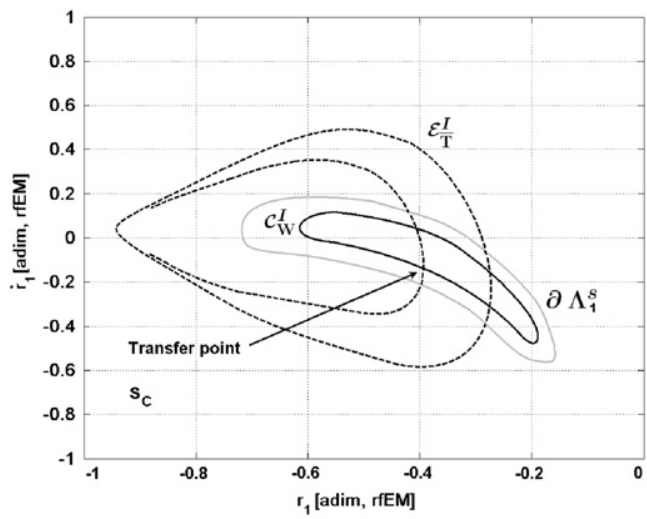

Fig. 6. Construction of the first guess interior transfer through the transfer point $\mathcal{W}^{I}=\mathcal{E}(I / \overline{\mathbf{T}})(t) \cap \mathcal{C}_{\mathbf{w}}^{I}(-t)$. (a) Final DPO and stable manifold construction. (b) $\mathcal{E}(I / \overline{\mathbf{T}})(t)$ and $\mathcal{C}_{\mathbf{W}}^{I}(-t)$ sets.

a

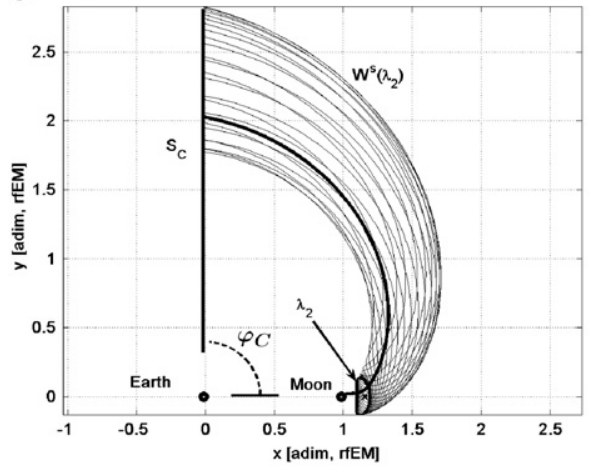

b

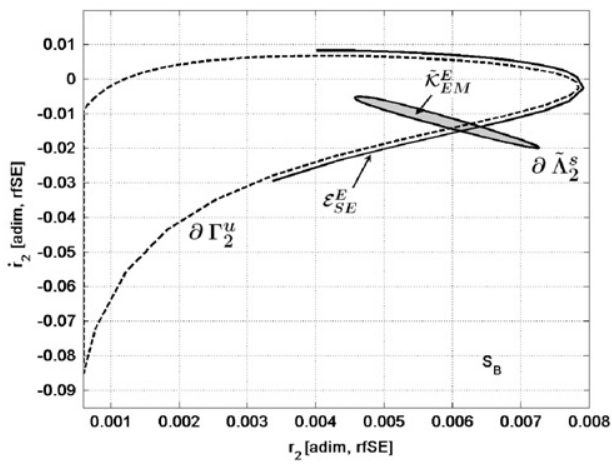

Fig. 7. Stable manifold $W^{s}\left(\lambda_{2}\right)$ and its section curve $\partial \tilde{\Lambda}_{2}^{s}$. The latter is used to define the set of orbits that lead to Moon capture $\left(\tilde{\mathcal{K}}_{E M}^{E}\right)$. In (a), the bold line shows a sample Moon capture trajectory. (a) Exterior branch of $W^{s}\left(\lambda_{2}\right)$. (b) $\mathcal{E}_{S E}^{E}$ and $\tilde{\mathcal{K}}_{E M}^{E}$ sets.

The stable manifold orbit in Eq. (17) can be expressed as

$\gamma_{\mathbf{w}}\left(\mathbf{y}_{f},-t\right)=\left\{\phi\left(\mathbf{y}_{f} \pm \delta \mathbf{y}_{s}, t_{i} ;-\tau\right) \mid-\tau \geq-t\right\}$

where $\mathbf{y}_{s}$ is the local stable eigenvector and $\delta$ is a small number (the \pm ambiguity is solved by choosing the sign that generates the interior branch of the manifold). Since the first part of the transfer is defined on $\mathcal{E}(I / \overline{\mathbf{T}})(t)$, lowthrust trajectories to DPO are contained in the set

$\mathcal{W}^{I}=\mathcal{E}_{\overline{\mathbf{T}}}^{I}(t) \cap \mathcal{C}_{\mathbf{W}}^{I}(-t)$

\subsection{Exterior capture}

In the EM model, by fixing $C_{E M} \lesssim C_{2}$, both $\lambda_{1}$ and $\lambda_{2}$ exist. The stable manifold $W^{s}\left(\lambda_{2}\right)$ is considered to approach the Moon from the exterior (see Fig. 7(a)). The stable manifold associated with $\lambda_{2}, W^{s}\left(\lambda_{2}\right)$, is computed starting from $\lambda_{2}$ and integrating backward until a certain surface of section is reached. Section $S_{C}$, making an angle $\varphi_{C}$ (counterclockwise) with the $x$-axis and passing through the Earth, is considered to cut $W^{s}\left(\lambda_{2}\right)\left(\varphi_{C}=\pi / 2\right.$ in Fig. $7(\mathrm{a})$ ). The corresponding section curve, $\partial \Lambda_{2}^{s}$ (computed on the $\left(r_{1}, \dot{r}_{1}\right)$-plane), is diffeomorphic to a circle. The set $\mathcal{K}_{E M}^{E}=\dot{\Lambda}_{2}^{s}$ is defined, where $\dot{\Lambda}_{2}^{s} \in S_{C}$ is the set of points inside $\partial \Lambda_{2}^{s}$, that leads to the Moon capture.

The set $\mathcal{K}_{E M}^{E}$ is defined on section $S_{C}$ (EM model). However, it is possible to represent $\mathcal{K}_{E M}^{E}$ on $S_{B}$ (SE model) through the transformation $\tilde{\mathcal{K}}_{E M}^{E}=\mathcal{M}\left(\mathcal{K}_{E M}^{E}\right)$. The operator $\mathcal{M}$ maps states on $S_{C_{E}}$ (EM model) to states on $S_{B}$ (SE model). In Fig. 7(b), $\tilde{\mathcal{K}}_{E M}^{E}$ and $\partial \tilde{\Lambda}_{2}^{s}$ are reported. Ballistic low energy Earth-Moon transfers are defined by $\mathcal{E}_{S E}^{E} \cap$ $\tilde{\mathcal{K}}_{E M}^{E}$ (see Mingotti et al. [15] for details).

Let us consider a g-type DPO about the Moon, with $J=3.00002258271$ in the EM system (see Fig. 8). The idea is to achieve Moon capture by using its exterior stable manifold. The transfer ends when the spacecraft reaches a point of the DPO. In analogy with Section 5.1, the final state of the transfer, $\mathbf{y}_{f}$, can be defined by

$\mathbf{y}_{f}=\mathbf{y}\left(\tau_{M}\right)=\phi\left(\mathbf{y}_{i}, 0 ; \tau_{M}\right)$.

The domain of admissible final states is then written as follows:

$\mathcal{Y}^{M}=\left\{\mathbf{y}_{f}\left(\tau_{M}\right) \mid \tau_{M} \in\left[0, P_{D P O}\right]\right\}$, 
a

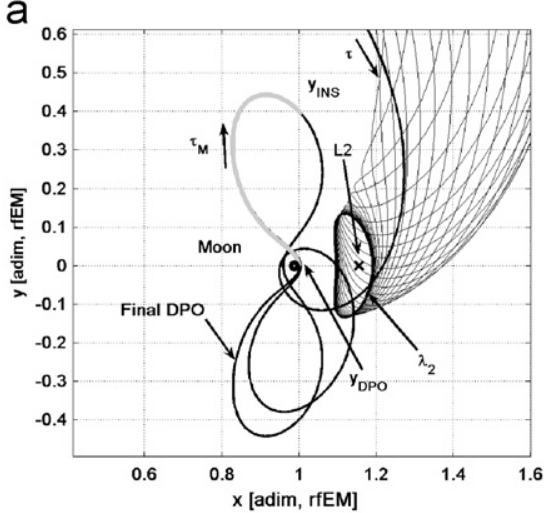

b

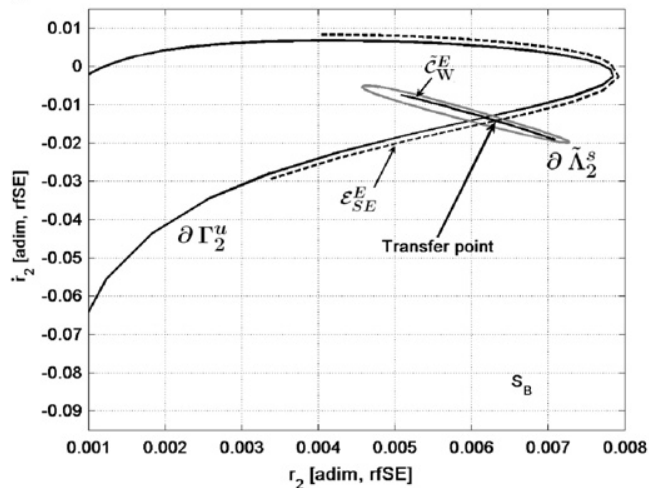

Fig. 8. The first guess exterior stable manifold capture solution as the transfer point $\mathcal{W}^{E}=\mathcal{E}_{S E}^{E} \cap \tilde{\mathcal{C}}_{\mathbf{W}}^{E}(-t)$, the latter reported on section $S_{B}$ in (b). (a) Stable manifold capture trajectory. (b) $\mathcal{E}_{S E}^{E}$ and $\tilde{\mathcal{C}}_{\mathbf{W}}^{E}(-t)$ sets.

a

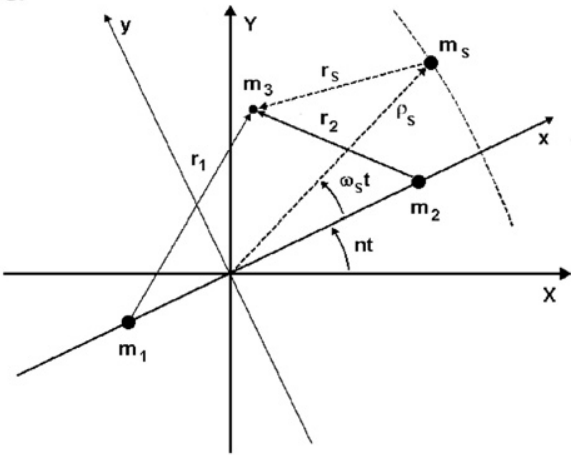

b
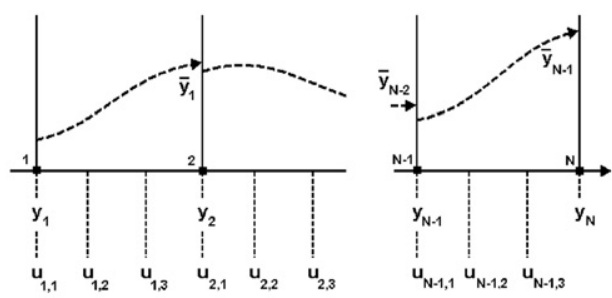

Fig. 9. Optimization process, dynamical model, and integration scheme. (a) Sun-perturbed Earth-Moon problem. (b) Direct multiple shooting scheme.

and the attainable set, for some $t \geq 0$ (i.e., $-t$ is a backward integration), containing stable manifold trajectories is

$\mathcal{C}_{\mathbf{W}}^{E}(-t)=\bigcup_{\mathbf{y}_{f} \in \mathcal{Y}^{M}} \gamma_{\mathbf{w}}\left(\mathbf{y}_{f}\left(\tau_{M}\right),-t\right)$

Each stable manifold orbit that leads to the final DPO around the Moon proposed in Eq. (22), at time $-t$, can be expressed as

$\gamma_{\mathbf{w}}\left(\mathbf{y}_{f},-t\right)=\left\{\phi\left(\mathbf{y}_{f} \pm \delta \mathbf{y}_{s}, t_{i} ;-\tau\right) \mid-\tau \geq-t\right\}$

where $\mathbf{y}_{s}$ is the local stable eigenvector and $\delta$ is a small number. The \pm ambiguity is solved by choosing the sign that produces the exterior branch. Since the first part of the transfer is defined on $\mathcal{E}_{S E}^{E}$, single-impulse Earth-Moon transfers are generated by

$\mathcal{W}^{E}=\mathcal{E}_{S E}^{E} \cap \tilde{\mathcal{C}}_{\mathbf{W}}^{E}(-t)$,

where $\tilde{\mathcal{C}}_{\mathbf{W}}^{E}(-t)=\mathcal{M}\left(\mathcal{C}_{\mathbf{W}}^{E}(-t)\right)$ (see Fig. 8(b)).

\section{Trajectory optimization}

First guess solutions found with either Eq. (19) or Eq. (24) are optimized into a four-body framework. The model used to take into account low-thrust propulsion and the gravitational attractions of the Sun, the Earth, and the Moon is [25]

$\ddot{x}-2 \dot{y}=\frac{\partial \Omega_{S}}{\partial x}+\frac{T_{x}}{m}, \quad \ddot{y}+2 \dot{x}=\frac{\partial \Omega_{S}}{\partial y}+\frac{T_{y}}{m}, \quad \dot{\theta}=\omega_{S}$,

$\dot{m}=-\frac{T}{I_{s p} g_{0}}$,

where

$\Omega_{S}(x, y, \theta)=\Omega\left(x, y, \mu_{E M}\right)+\frac{m_{S}}{r_{S}}-\frac{m_{S}}{\rho_{S}^{2}}(x \cos \theta+y \sin \theta)$,

and

$r_{S}=\left[\left(x-\rho_{S} \cos \theta\right)^{2}+\left(y-\rho_{S} \sin \theta\right)^{2}\right]^{1 / 2}$.

See Fig. 9(a) and Mingotti et al. [15,20,22] for details on this model.

The controlled dynamics (25) is written in the firstorder form

$\dot{x}=v_{x}$,

$\dot{y}=v_{y}$,

$\dot{v}_{x}=2 v_{y}+\Omega_{S x}+T_{x} / m$,

$\dot{v}_{y}=-2 v_{x}+\Omega_{S y}+T_{y} / m$, 
$\dot{\theta}=\omega_{S}$,

$\dot{m}=-T /\left(I_{s p} g_{0}\right)$,

with $v_{x}=\dot{x}$ and $v_{y}=\dot{y}$. In a compact explicit form, system (28) reads

$\dot{\mathbf{y}}=\mathbf{f}[\mathbf{y}(t), \mathbf{T}(t), \mathbf{p}, t]$,

where $\mathbf{f}$ is the vector field, $\mathbf{T}=\left\{T_{x}, T_{y}\right\}^{\top}$ is the thrust vector, the state vector is $\mathbf{y}=\left\{x, y, v_{x}, v_{y}, \theta, m\right\}^{\top}$, and $\mathbf{p}$ is a vector of parameters. The aim is finding the guidance law $\mathbf{T}=\mathbf{T}(t), t \in\left[t_{i}, t_{f}\right]$, that minimizes a prescribed scalar performance index

$J=J(\mathbf{y}, \mathbf{T}, \mathbf{p}, t)$,

while satisfying certain mission constraints.

\subsection{Numerical scheme}

The optimal control problem is transcribed into a nonlinear programming (NLP) problem and solved with a multiple shooting scheme [1]. The main idea is to divide the trajectory into $N-1$ intervals. Thus, the time domain is discretizes as

$t_{i}=t_{1}<t_{2}<\cdots<t_{N-1}<t_{N}=t_{f}$,

and the NLP vector if variables is

$\mathbf{x}=\left\{\mathbf{v}_{1}, \mathbf{v}_{2}, \ldots, \mathbf{v}_{N-1}, \mathbf{v}_{N}, t_{i}, t_{f}, \mathbf{p}\right\}^{\top}$,

where $\mathbf{v}$ is a vector containing the states and the controls (see below). The initial value of each trajectory segment, $\mathbf{v}_{j}$, is propagated forward under Eq. (28), from $t_{j}$ to $t_{j+1}$, $j=1, \ldots, N-1$. In this work, a fixed step Runge-KuttaFehlberg scheme of the eighth order is implemented. The respect of the dynamics is obtained by enforcing

$\boldsymbol{\eta}_{j}=\overline{\mathbf{v}}_{j}-\mathbf{v}_{j+1}=0$,

where $\overline{\mathbf{v}}_{j}$ is the result of the integration within the $j$-th interval. The complete constraint vector is assembled in the following form:

$\mathbf{c}(\mathbf{x})=\left\{\boldsymbol{\psi}_{i}\left[\mathbf{v}\left(t_{1}\right), t_{1}, \mathbf{p}\right], \boldsymbol{\eta}_{1}, \ldots, \boldsymbol{\eta}_{N-1}, \boldsymbol{\psi}_{f}\left[\mathbf{v}\left(t_{N}\right), t_{N}, \mathbf{p}\right]\right\}^{\top}$,

where $\psi_{i}$ and $\psi_{f}$ are generic initial and final boundary nonlinear conditions. In the same way, the performance index is expressed as a function of the NLP variables; i.e., $J=J(\mathbf{x})$.

According to the direct approach followed in this paper, the quantities $\mathbf{v}$ are made up by the state $\mathbf{y}$ and the control $\mathbf{u}$. The control law is expressed within each interval by using a second level time grid [6]. Let the time domain be divided into a two-level grid: the main $N$ nodes grid, as described before, and the second level subdivision of each $N-1$ intervals into $M-1$ subsegments, as shown in Fig. 9(b). According to this formulation, the sates $\mathbf{y}_{j}$ of the problem are associated with each $j=1, \ldots, N$ grid node; as for the controls, they are organized in a vector with the form $\mathbf{u}_{j, k}$. The first subscript $j$ stands for the main level grid, while the second $k=1, \ldots, M-1$ subscript represents the second level subdivision for each $j$-node. In this way, a third-order spline interpolation of the controls is assumed within each main level interval, when $M=4$ is chosen.

\subsection{Low-thrust problem statement}

In low-thrust, interior transfers, the vector of variables is $\mathbf{x}=\left\{(\mathbf{y}, \mathbf{T})_{1}, \ldots,(\mathbf{y}, \mathbf{T})_{N}, t_{1}, t_{N}, \mathbf{p}\right\}^{\top}$.

Although the first guess control law is aligned with the synodic velocity of the spacecraft (tangential thrust of magnitude $\bar{T}$ ), the optimization processes all the variables in Eq. (35), and therefore it is free to modify the control direction and magnitude.

The spacecraft is assumed to be initially on a GTO about the Earth. The initial state, $\mathbf{y}_{1}$, corresponds to the periapsis of such orbit. As both eccentricity and apsidal altitudes are prescribed, this initial state depends only upon the argument of perigee $\omega_{E}$. The initial boundary condition is

$\psi_{i}=\mathbf{y}_{1}-\mathbf{y}_{i}\left(\omega_{E}\right)=0$.

The transfer ends when the spacecraft reaches any point of the prescribed DPO. The final state is defined by Eq. (15), and therefore the final boundary condition reads

$\psi_{f}=\mathbf{y}_{N}-\phi\left(\mathbf{y}_{i}, 0 ; \tau_{M}\right)=0$.

The two scalars $\tau_{M}$ and $\omega_{E}$ define the vector of parameters $\mathbf{p}$. These are taken as variables in Eq. (35) to find their optimal values. The nonlinear equality constraint vector is made up by the boundary conditions and the integration defects

$\mathbf{c}(\mathbf{x})=\left\{\boldsymbol{\psi}_{i}, \boldsymbol{\eta}_{1}, \ldots, \boldsymbol{\eta}_{N-1}, \boldsymbol{\psi}_{f}\right\}^{\top}$.

To avoid collision with the two primaries, the following inequality constraints are imposed:

$\boldsymbol{\Psi}_{j}^{c}\left(\mathbf{y}_{j}\right):=\left\{\begin{array}{l}R_{E}^{2}-\left(x_{j}+\mu\right)^{2}-y_{j}^{2} \leq 0 \\ R_{M}^{2}-\left(x_{j}-1+\mu\right)^{2}-y_{j}^{2} \leq 0, \quad j=2, \ldots, N-1 .\end{array}\right.$

The quantities $R_{E}$ and $R_{M}$ are the radius of the Earth and the Moon, respectively. The flight time has to be positive, $\boldsymbol{\Psi}^{t}=t_{1}-t_{N} \leq 0$

Finally, another inequality constraint is imposed along the whole transfer to model the saturation of the low-thrust engine,

$\boldsymbol{\Psi}_{j}^{s}\left(\mathbf{T}_{j}\right)=T_{j}-T_{\max } \leq 0, \quad j=1, \ldots, N$,

where $T_{\max }=0.5 \mathrm{~N}$. The complete inequality constraint vector is

$\mathbf{g}(\mathbf{x})=\left\{\boldsymbol{\Psi}_{1}^{s}, \boldsymbol{\Psi}_{2}^{c}, \boldsymbol{\Psi}_{2}^{s}, \ldots, \boldsymbol{\Psi}_{N-1}^{c}, \boldsymbol{\Psi}_{N}^{s}, \boldsymbol{\Psi}^{t}\right\}^{\top}$.

The performance index to minimize is

$J=\int_{t_{i}}^{t_{f}} \frac{T(t)}{I_{s p} g_{0}} \mathrm{~d} t$

that corresponds to the propellant mass, $m_{p}=m_{i}-m\left(t_{f}\right)$, needed to perform the transfer. The optimization problem for the low-thrust interior transfers is defined by

$\min _{\mathbf{x}} J(\mathbf{x}) \quad$ subject to $\begin{aligned} & \mathbf{c}(\mathbf{x})=0, \\ & \mathbf{g}(\mathbf{x}) \leq 0 .\end{aligned}$

\subsection{Single-impulse problem statement}

In single-impulse, exterior transfers the variable vector is $\mathbf{x}=\left\{(\mathbf{y}, \mathbf{W})_{1}, \ldots,(\mathbf{y}, \mathbf{W})_{N}, t_{1}, t_{N}, \mathbf{p}\right\}^{\top}$, 
where $\mathbf{W}=\{0,0\}^{\top}$; i.e., a ballistic transfer is a "special" lowthrust transfer with zero thrust.

The equality constraint vector is constructed in the same way as Eq. (38), with the exception of the initial condition. In this case, the transfer begins from a circular parking orbit around the Earth. The initial boundary conditions are therefore

$\psi_{i}\left(\mathbf{y}_{1}, t_{1}\right):=\left\{\begin{array}{l}\left(x_{1}+\mu\right)^{2}+y_{1}^{2}-r_{i}^{2}=0, \\ \left(x_{1}+\mu\right)\left(\dot{x}_{1}-y_{1}\right)+y_{1}\left(\dot{y}_{1}+x_{1}+\mu\right)=0,\end{array}\right.$

which forces the initial state to belong to a circular orbit of radius $r_{i}=R_{E}+h_{E}$; the final condition reads as stated in Eq. (37). As for the inequality constraint vector, this is defined in the same way as in Eq. (42), with the constraint in Eq. (41) that is automatically satisfied.

The performance index to minimize is the initial velocity variation

$J(\mathbf{x})=\Delta v_{1}=\sqrt{\left(\dot{x}_{1}-y_{1}\right)^{2}+\left(\dot{y}_{1}+x_{1}+\mu\right)^{2}}-v_{i}$,

where $v_{i}$ is the velocity on the circular parking orbit. The optimization problem for the single-impulse, exterior transfers is stated in the same fashion of Eq. (44).

\subsection{Hints on numerical implementation}

All numerical integrations have been carried out with a Runge-Kutta-Fehlberg scheme with absolute and relative tolerances set to $10^{-10}$. This tolerance is deemed sufficient to accurately describe close Moon encounter (no regularization of collisions is implemented). In the definition of the first guess solutions, Eqs. (19) and (24), small discontinuities are tolerated. Solutions whose distance is in the range $\left[10^{-6}-10^{-4}\right]$ are admitted to the optimization; this tolerance guarantees the convergence of the subsequent optimization. Four first guesses have been optimized for the two trajectory cases considered. The optimization problems have been solved with a sequential quadratic programming scheme implementing an active-set strategy with tolerance on equality and inequality constraints set to $10^{-10}$. The gradient of the objective function as well as the Jacobians of the equality and inequality constraints are calculated numerically. Once an optimal solution is found, it is assessed a posteriori by forward integrating the optimal initial condition under a Runge-Kutta-Fehlberg scheme of the eighth order, and by cubic interpolation of the discrete optimal control solution.

In low-thrust transfers $N=81$ and $M=4$ are chosen. This means that the complete trajectories are divided into 80 uniform main intervals and into 320 uniform second level subsegments. This leads 1204 variables (considering $\left.\mathbf{p}=\left\{\omega_{E}, \tau_{M}\right\}\right), 82$ equality constraints, and 162 inequality constraints. The average computational time for this kind of problem is a few hours on a standard desktop pc.

In single-impulse transfers $N=41$ and $M=4$ are chosen. This having 40 first level intervals and 160 second level subsegments. This leads to 243 variables (considering $\mathbf{p}=\left\{\tau_{M}\right\}$ ), 42 equality constraints, and 82 inequality constraints. The average computational time for this kind of problem is a few tens of minutes on a standard desktop pc.

\section{Optimized transfer solutions}

Optimal solutions to distant prograde orbits around the Moon are shown. Two mission concepts are proposed: (a) low-thrust, stable-manifold interior transfers; (b) single-impulse, stable-manifold exterior transfers. Both examples are shown in the EM rotating (first guess and optimized solutions) and Earth-centered inertial frame (optimized solution only); see Figs. 10 and 12(a) for concept (a), Figs. 11 and 12(b) for concept (b). In both cases, the transfers terminate when the spacecraft reaches a point of the g-type DPO chosen (in the examples below the chosen DPO has the period equal to that of the Moon; i.e., 1:1 resonance).

The results are shown in Table 1 . The first two solutions (sol.1, sol.2) correspond to the low-thrust, stable
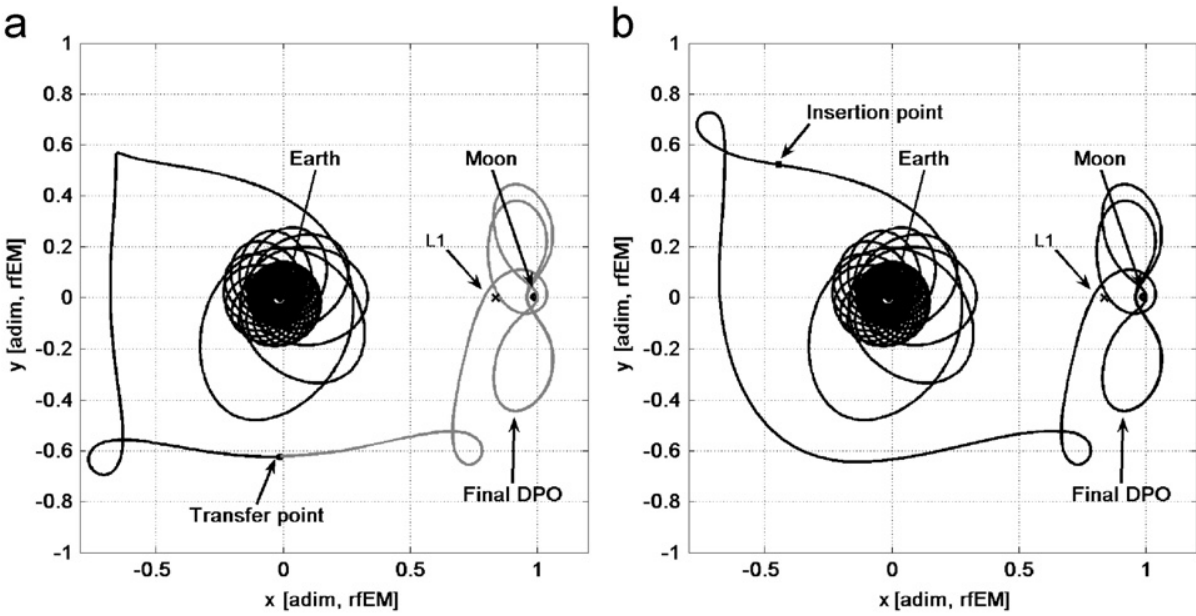

Fig. 10. Optimized low-thrust, stable-manifold transfer to a distant periodic orbit around the Moon. These figures correspond to sol.2 in Table 1. (a) First guess low-thrust, stable-manifold trajectory (rotating EM reference frame). (b) Optimized low-thrust, stable-manifold trajectory (rotating EM reference frame). 
a

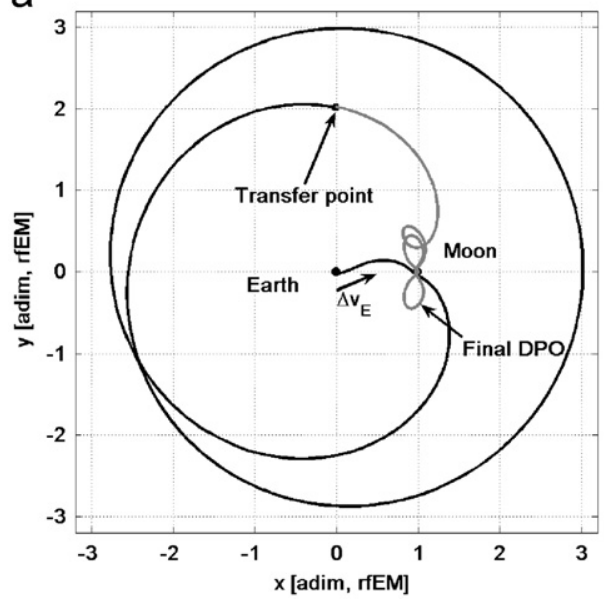

b

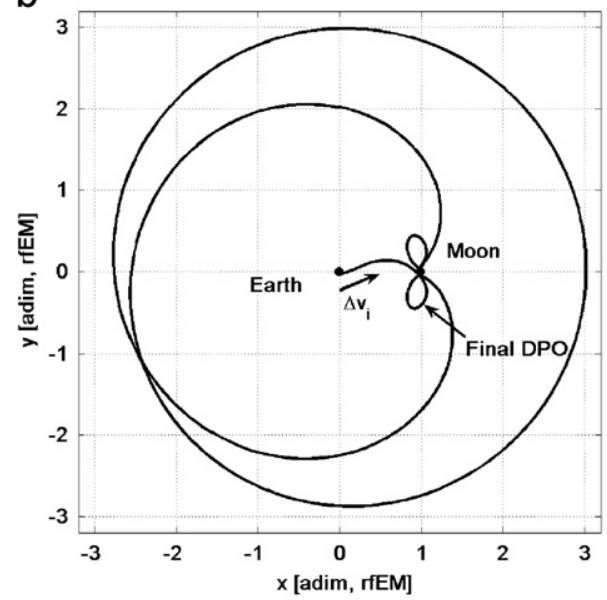

Fig. 11. Optimized single-impulse, stable-manifold transfer to a distant periodic orbit around the Moon. These figures correspond to sol.4 in Table 1 . (a) First guess single-impulse trajectory (rotating EM reference frame). (b) Optimized single-impulse trajectory (rotating EM reference frame).
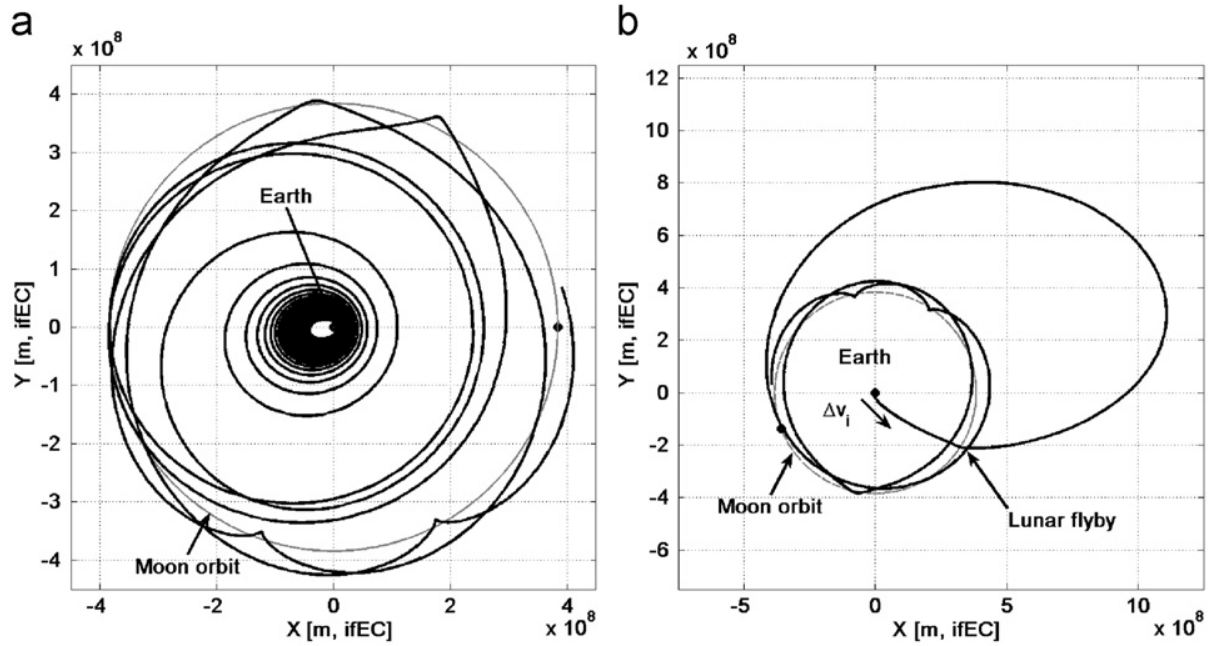

Fig. 12. Optimized low-thrust, stable-manifold and single-impulse, stable-manifold transfers to a distant periodic orbit around the Moon. These figures correspond to sol.2 and sol.4 in Table 1 and are drawn in the inertial geocentric reference frame. (a) Optimized low-thrust, stable-manifold trajectory (inertial geocentric reference frame). (b) Optimized single-impulse trajectory (inertial geocentric reference frame).

manifold transfers. The remaining two (sol.3, sol.4) represent the single-impulse, stable manifold transfers. These solutions are compared to reference interior transfers to the same final DPO [21]. The latter are obtained by targeting a piece of the interior stable manifold of DPO with a two-impulse strategy (see Mingotti et al. [21] for more details).

The second column of Table 1 represents the initial orbit. In the third column, $\Delta v_{i}$ stands for the magnitude of the initial impulsive maneuver. In the fourth column, $\Delta v_{f}$ represents the final impulsive maneuver that permits a stable permanent capture into the final orbit around the Moon. This term is not contemplated in our study as stable manifolds associated to the DPO are used (in [13] those of the Lyapunov orbits are considered).

In the fifth column of Table $1, f_{t}$ is the overall mass fraction necessary to carry out the transfer.
Table 1

Optimized transfers to lunar DPO. The transfers are compared to two reference, impulsive solutions (ref.1 and ref.2) having the same final DPO [21].

\begin{tabular}{lcccccc}
\hline Type & Origin & $\Delta v_{i}(\mathrm{~m} / \mathrm{s})$ & $\Delta v_{f}(\mathrm{~m} / \mathrm{s})$ & $f_{t}$ (adim.) & $\Delta v(\mathrm{~m} / \mathrm{s})$ & $\Delta t$ (days) \\
\hline sol.1 & GTO & - & - & 0.107 & 1446 & 125 \\
sol.2 & GTO & - & - & 0.105 & 1417 & 121 \\
sol.3 & LEO & 3161 & - & 0.658 & 3161 & 92 \\
sol.4 & LEO & 3154 & - & 0.657 & 3154 & 90 \\
ref.1 & LEO & 3166 & 603 & 0.722 & 3769 & 20 \\
ref.2 & LEO & 3138 & 748 & 0.733 & 3886 & 38 \\
\hline
\end{tabular}

For single-impulse transfers this is

$f_{t}=1-\exp \left(-\frac{\Delta v_{i}}{I_{s p}^{h t} g_{0}}\right)$, 
where $I_{s p}^{h t}=300 \mathrm{~s}$ is assumed for high-thrust, chemical engines. In case of low-thrust transfers $f_{t}$ is

$f_{t}=\frac{1}{m_{i}} \int_{t_{i}}^{t_{f}} \frac{T(t)}{I_{s p} g_{0}} \mathrm{~d} t$

where $I_{s p}^{l t}=3000 \mathrm{~s}$ is the specific impulse of low-thrust, electrical engines. An initial mass of $m_{i}=1000 \mathrm{~kg}$ is considered in Eq. (49). The term $\Delta v$ in the sixth column is the total cost of the transfer. For impulsive solutions, $\Delta v=\Delta v_{i}+\Delta v_{f} ; \quad$ for low-thrust solutions, $\Delta v=-I_{s p}^{l t} g_{0} \log \left(1-f_{t}\right)$. This indicator is useful to compare the low-thrust with impulsive solutions, although the dependency on the engine efficiency (i.e., $I_{s p}$ ) remains. The last column of Table 1 is the overall transfer time.

\subsection{Discussion of the results and possible applications}

By comparing Fig. 10(a) with Fig. 10(b) and Fig. 11(a) with Fig. 11(b), it can be noticed that the general shape of the transfer, as viewed in the rotating frame, is not subject to major variations when going from the first guess to the optimized solution. The optimal control step solves the discontinuity in the first guess and refines locally the trajectory by considering the perturbation of the Sun.

First of all, as far as it concerns the optimization algorithm, comparing Fig. 10(a) with Fig. 10(b) and Fig. 11(a) with Fig. 11(b), it is possible to notice that the general shape of the transfers changes just a little bit: the formulation of the optimal control problem, basically, solve the discontinuity in the first guess solutions and refine them considering also the gravitational attraction of the Sun.

Solutions sol.3 and sol.4 outperform those found in literature in terms of propellant mass consumption, while the transfer time is higher (starting from the same LEO and targeting the same DPO). Basically, in the singleimpulse transfers the savings are associated to a better exploitation of the invariant manifolds structure directly associated to the DPO.

As far as it concerns sol.1 and sol.2, a fair comparison is not possible as the initial conditions of the transfers are different: GTO for the low-thrust case and LEO for the impulsive case. Therefore, sol.1 and sol.2 are optimal in the class of low-thrust tangential transfers. Assuming a different control law (e.g., a variable-direction low-thrust) those results could be ameliorated.

About the $10 \%$ of the total mass is needed to reach the DPO about the Moon departing from a GTO. This is a little higher than the $9 \%$ needed to reach the $L 1$ and $L 2$ halos departing from the same orbit [14]. This occurs as the energy level of the DPO is higher than that of the halos. On the other side, while the halos remain at about the same distance to the Moon, the DPO offer the opportunity to perform close encounters with the Moon (which can be exploited, for instance, to deliver payloads on the Moon surface).

As for the impulsive case, the cost to reach the chosen DPO is equivalent to that needed to place the spacecraft into an exterior low-energy transfer [22]. However, in the case of transfers to DPO, in principle no maneuvers are needed to keep the spacecraft about the Moon (while a second maneuver is needed in low-energy transfers to stabilize the spacecraft about the Moon).

Special DPO families around the Moon have been studied. These show integer resonance with Moon's orbit, as well as integer relationships with the Moon's rotational motion. DPO around the Earth and around the Moon may have appealing properties from the practical point of view. Among them, DPO around the Earth offer the possibility of avoiding magnetic and radiation fields, and therefore they are useful for astrophysical applications. DPO around the Moon may be used by data-relay satellites to establish almost permanent communications with the far side of the Moon with only one spacecraft. DPO can also be exploited in formation flying applications [5]. In general, it is possible to devise many new-concept space mission applications involving DPO [18].

\section{Conclusions}

In this paper two different techniques to design Earthto-Moon transfers have been investigated. Both techniques exploit the stable manifold of the distant periodic orbits about the Moon. It is shown that such orbits are accessible with either interior or exterior transfers, as well as with either high- and low-thrust propulsion. Optimized solutions are obtained in the framework of an optimal control problem. The results show reduced costs and moderate flight times.

Alike periodic orbits around the collinear equilibrium points of the restricted three-body problem, distant periodic orbits present a rich phase-space structure. However, the latter offer the advantage of orbiting around the Moon too, and therefore they are suitable for many applications where halo or Lissajous orbits cannot be used. The stable manifolds of distant periodic orbits lead to a permanent capture stage about the Moon. This feature has high potentialities, and may be exploited to devise novel space mission concepts.

\section{References}

[1] J.T. Betts, Survey of numerical methods for trajectory optimization, J. Guid. Control Dyn. 21 (2) (1998) 193-207.

[2] A. Celletti, L. Stefanelli, E. Lega, C. Froeschle, Global dynamics of the regularized restricted three-body problem with dissipation, Celestial Mech. Dyn. Astron. 109 (2011) 265-284.

[3] C. Conley, Low energy transit orbits in the restricted three-body problem, SIAM J. Appl. Math. 16 (1969) 732-746.

[4] M. Dellnitz, O. Junge, M. Post, B. Thiere, On target for Venus: set oriented computation of energy efficient low thrust trajectories, Celestial Mech. Dyn. Astron. 95 (2006) 357-370.

[5] J. Demeyer, P. Gurfil, Transfer to distant retrograde orbits using manifold theory, J. Guid. Control Dyn. 30 (5) (2007) 1261-1273.

[6] P.J. Enright, B.A. Conway, Discrete approximations to optimal trajectories using direct transcription and nonlinear programming, Control Dyn. 15 (1992) 994-1002.

[7] G. Gomez, A. Jorba, J. Masdemont, C. Simo, Study of the transfer from the Earth to a halo orbit around the equilibrium point L1, Celestial Mech. Dyn. Astron. 56 (1993) 239-259.

[8] G. Gomez, W.S. Koon, M.W. Lo, J.E. Marsden, J. Masdemont, S.D. Ross, Invariant manifolds, the spatial three-body problem and space mission design, Adv. Astronaut. Sci. 109 (2001) 3-22.

[9] M. Hénon, Numerical exploration of the restricted problem. V, Astronaut. Astrophys. 1 (1969) 223-238. 
[10] K.C. Howell, B.T. Barden, M.W. Lo, Application of dynamical systems theory to trajectory design for a libration point mission, J. Astronaut. Sci. 45 (2) (1997) 161-178.

[11] W. Koon, M. Lo, J. Marsden, S. Ross, Low energy transfer to the Moon, Celestial Mech. Dyn. Astron. 81 (2001) 63-73.

[12] J. Llibre, R. Martinez, C. Simo, Transversality of the invariant manifolds associated to the Lyapunov family of periodic orbits near $L_{2}$ in the restricted three-body problem, J. Differ. Equat. 58 (1985) 104-156.

[13] X. Ming, X. Shijie, Exploration of distant retrograde orbits around Moon, Acta Astronaut. 65 (5-6) (2009) 853-860.

[14] G. Mingotti, F. Topputo, F. Bernelli-Zazzera, Combined optimal lowthrust and stable-manifold trajectories to the Earth-Moon halo orbits, in: American Institute of Physics Conference Proceedings, vol. 886, 2007, pp. 100-110.

[15] G. Mingotti, F. Topputo, F. Bernelli-Zazzera, Low-energy, low-thrust transfers to the Moon, Celestial Mech. Dyn. Astron. 105 (1-3) (2009) 61-74.

[16] G. Mingotti, F. Topputo, F. Bernelli-Zazzera, Numerical methods to design low-energy, low-thrust Sun-perturbed transfers to the Moon, in: Proceedings of the 49th Israel Annual Conference on Aerospace Sciences, 2009, pp. 1-14.

[17] G. Mingotti, Trajectory Design and Optimization in Highly Nonlinear Astrodynamics, Ph.D. Dissertation, Politecnico di Milano, 2010.

[18] G. Mingotti, P. Gurfil, Mixed low-thrust invariant-manifold transfers to distant prograde orbits around Mars, J. Guid. Control Dyn. 33 (2011) 1753-1764
[19] G. Mingotti, F. Topputo, F. Bernelli-Zazzera, Earth-Mars transfers with ballistic escape and low-thrust capture, Celestial Mech. Dyn. Astron. 110 (2011) 169-188.

[20] G. Mingotti, F. Topputo, F. Bernelli-Zazzera, Optimal low-thrust invariant manifold trajectories via attainable sets, J. Guid. Control Dyn. 34 (2011) 1644-1655.

[21] G. Mingotti, F. Topputo, Ways to the Moon: a survey, Adv. Astronaut. Sci. 140 (2011) 2531-2548.

[22] G. Mingotti, F. Topputo, F. Bernelli-Zazzera, Efficient efficient invariant-manifold, low-thrust planar trajectories to the Moon, Commun. Nonlinear Sci. Numer. Simulation 17 (2012) 817-831.

[23] C.A. Ocampo, G.W. Rosborough, Transfer Trajectories for Distant Retrograde Orbiters of the Earth, NASA STI/RECON Technical Report A, 95, 1993.

[24] J.S. Parker, M.W. Lo, Unstable resonant orbits near Earth and their applications in planetary missions, in: Proceedings of the 17 th AIAA/AAS Conference, vol. 1, 2007, pp. 1-27.

[25] C. Simo, G. Gomez, A. Jorba, J. Masdemont, The bicircular model near the triangular libration points of the RTBP, in: From Newton to Chaos, 1995, pp. 343-370.

[26] V. Szebehely, Theory of Orbits: The Restricted Problem of Three Bodies, Academic Press, New York, 1967.

[27] R. Thurman, P.A. Worfolk, The Geometry of Halo Orbits in the Circular Restricted Three-Body Problem, Geometry Center Research Report, University of Minnesota, GCG95, 1996. 